\title{
Simulating the processes of science, technology, and innovation
}

\author{
Guest Editors:
}

Katy Börner, Bruce Edmonds, Staša Milojević and Andrea Scharnhorst. 